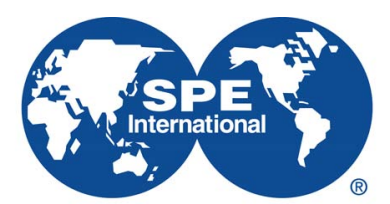

Society of Petroleum Engineers

\title{
SPE-185922-MS
}

\section{Petrophysical Well Log Analysis through Intelligent Methods}

\author{
Leili Moghadasi, Ehsan Ranaee, Fabio Inzoli, and Alberto Guadagnini, Politecnico di Milano \\ Copyright 2017, Society of Petroleum Engineers \\ This paper was prepared for presentation at the SPE Bergen One Day Seminar held in Bergen, Norway, 5 April 2017.
}

This paper was selected for presentation by an SPE program committee following review of information contained in an abstract submitted by the author(s). Contents of the paper have not been reviewed by the Society of Petroleum Engineers and are subject to correction by the author(s). The material does not necessarily reflect any position of the Society of Petroleum Engineers, its officers, or members. Electronic reproduction, distribution, or storage of any part of this paper without the written consent of the Society of Petroleum Engineers is prohibited. Permission to reproduce in print is restricted to an abstract of not more than 300 words; illustrations may not be copied. The abstract must contain conspicuous acknowledgment of SPE copyright.

\section{Abstract}

This work is focused on exploring the applicability of intelligent methods in assessing porosity and permeability in the context of reservoir characterization. The main motivation underlying our study is that appropriate estimation of reservoir petrophysical parameters such as porosity and/or permeability is a key step for in-situ hydrocarbon reservoir evaluation. We ground our analysis on information on logdepth, caliper, conductivity, sonic logging, natural gamma, density and neutron porosity, water saturation, percentage of shale volume, and type of lithology collected from well loggings in an oil field in the middleeast (a total number of 11 exploratory wells are considered). Data also include porosities and permeabilities evaluated on core samples from the same wells. All these data are embedded in a neural network-based approach which enables us to establish input-output relationships in terms of an optimized number of input variables. Three diverse intelligent techniques are tested. These include: $(i)$ classical artificial neural networks; (ii) artificial neural networks based on principal component analysis (PCA) transformation; and (iii) statistical neural networks based on a bagging approach. Our results suggest that the statistical neural network is most effective for the field setting considered. The application of this neural network with 9 input parameters provides reliable performances in $94 \%$ and $81 \%$ of the cases, respectively in the training and validation phases, for the estimation of porosity. A trained network with 10 input parameters leads to successfull reproduction of permeability values in $85 \%$ and $79.5 \%$ of the cases, respectively during training and validation of the network. Results from this study are expected to be transferable to applications involving evaluation of petrophysical properties of a target reservoir in the presence of incomplete well $\log$ datasets.

\section{Introduction}

Petrophysical propetises (such as porosity and permeability) are critical variables for the characterization of hydrocarbon reservoirs. In general, characterization of porosity and permeability may be assessed through the analysis of core samples, well logging, well testing, field production data and seismic information. Data collected from well logging are frequently available to provide valuable (albeit indirect) information about mineralogy, texture, sedimentary structures and fluid content of a reservoir (e.g., Wong et al., 2000 and references therein). There are also some empirical models available to infer porosity and permeability from well $\log$ data upon relying on a variety of input information (e.g., Coates and Dumanoir, 1973; Johnson, 1994). The development of modern well logging tools, techniques and systems has favored 
the use of such empirical models, which involve a reduced cost and time investment when compared to conventional coreflooding experiments. In this context, regression techniques are then typically applied to project available core plug datasets onto unsampled locations in the reservoir and then employ the ensuing results to estimate permeability and porosity. The quality of these estimates is strongly affected by the degree of system heterogeneity and by the ability of the available sample plugs to be representative of the spatial distribution of the target petrophysical characteristics. Outcomes of such an analysis are typically applicable solely for the characterization of the wells where cores and plugs are taken from (Ligtenberg and Wansink, 2001).

Statistical approaches and intelligent techniques such as artificial neural networks (ANN) have been proposed as versatile tools to deal with estimations of rock petrophysical characteristics (Wong et al., 2000). Some recent investigations suggest that ANNs can be superior to other regular statistical methods in predicting porosity and permeability from well logging data (e.g., Mohaghegh, 2005; Lim, 2005; Aminian et al., 2005). ANN is a nonparametric tool which has become increasingly popular to analyze and interpret well logging data in the presence of incomplete information. ANNs are essentially translated into computer codes attempting to mimic biological learning processes of the human nervous system (Huang et al., 1996; Huang and Williamson, 1997). One of the main capabilities of ANNs is pattern recognition, due to their capability of assimilating the nature of the dependency between input and output variables of a target process. Since learning is essentially based on pattern recognition, a trained network classifies learned patterns and provides predictions of desired outputs (Mohaghegh, 2000). ANN approaches are attractive mainly because (i) there is no need to completely analyze the mathematical formulation rendering the physical processes governing a system evolution and (ii) they are essentially free from linearity assumptions for the interpolation of the quantities of interest (Helle et al., 2001). We rely here on ANNs and take advantage of their ability to serve as a nonlinear regression technique to transfer at unsampled locations information collected at a selected set of well logging from standard core analyses.

We analyze the ability of three diverse intelligent techniques to provide estimates of porosity and permeability of an oil reservoir in the middle-east. These techniques include $(i)$ classical artificial neural networks (ANNs); (ii) artificial neural networks based on principal component analysis (PCA); and (iii) statistical neural networks based on bagging technique. For all of the employed techniques, we design and train a back-propagation neural network (BPNN) for the reproduction of porosity and permeability based on data collected from well logging. The optimum trained network is then applied to predict relevant reservoir properties at wells with incomplete/missed logging information.

Defining input variables is critical to obtain appropriate predictions of the desired outputs from ANNs. Selecting input variables governing the process considered may be challenging in the presence of $(i)$ a large number of potential input variables; (ii) correlations between these, which can give rise to redundancy; and (iii) some variables which are practically not influential to the target process. Preliminary statistical analyses are then useful, because they assist to identifying the occurrence of such issues.

It is expected that the quality and efficiency of the training of a network can increase through the application of targeted pre-processing statistical analyses to the available dataset, such as principal component analysis (PCA). The latter enables us to highlight input/output characteristic features needed to design an efficient ANN (see, e.g., Olsson et al., 2004; Gibbs et al., 2006; and Bowden, 2003 for additional details). Ranaee et al. (2014) show that (linear/non-linear) PCA techniques can be considered as a viable pre-processing step to conveniently decrease the complexity of the system by reducing the dimensionality of the input variable space and improve the efficiency of training ANNs while preserving the accuracy of the ensuing results.

In the following we present a summary of the techniques we employ to evaluate prosity and permeability as target quantities of interest as well as their applications to the field setting considered. 


\section{Materials and methods}

Artificial Neural networks (ANNs) have been successfully used in a variety of petroleum engineering applications, including reservoir characterization and optimization of field operations (Mohaghegh, 2000; Tamhane et al., 2000; Lim et al., 2004). ANNs can (in principle) learn and mimic underlying relationships between a provided set of input and output (desired) data. A trained network is then capable of classifying a newly emerging pattern between input-output and providing system responses based on the knowledge acquired from previously introduced/acquired patterns. A trained ANN can then be used as a surrogate for a mathematical model which is either unknown or known but associated with a marked degree of complexity (Silpngarmlers et al., 2002). These surrogate models have the added value of being able to capture nonlinearities of relationships between variables (e.g., Ranaee et al., 2014 and references therein). Hence, the performance of the neural network critically depends on the reliability of the training process.

Figure 1 depicts a schematic three-layer ANN system. In general, one can resort to $(i)$ supervised or (ii) unsupervised techniques to train a network (Mohaghegh, 2000). We employ here a supervised backpropagation algorithm (BPNN) which is the most widely used ANN training algorithm (Haykin, 1999; Lim, 2003; Aminian and Ameri, 2005). The available dataset is subdivided into sub-sets which are then employed for ( $i$ ) training, (ii) validation, and (iii) as a test bed for the neural network. A BPNN training process includes feeding training samples as input vectors to the network, calculating prediction errors (e.g., in terms of mean square error, MSE) with respect to measured values of the desired model output, and then tuning the weights of the network to minimize such errors (Chen, 2006). In this study we employ the Levenberg-Marquardt optimization algorithm for the training of the system (Hagan and Menhaj, 1994). The BPNN is based on the following formulation

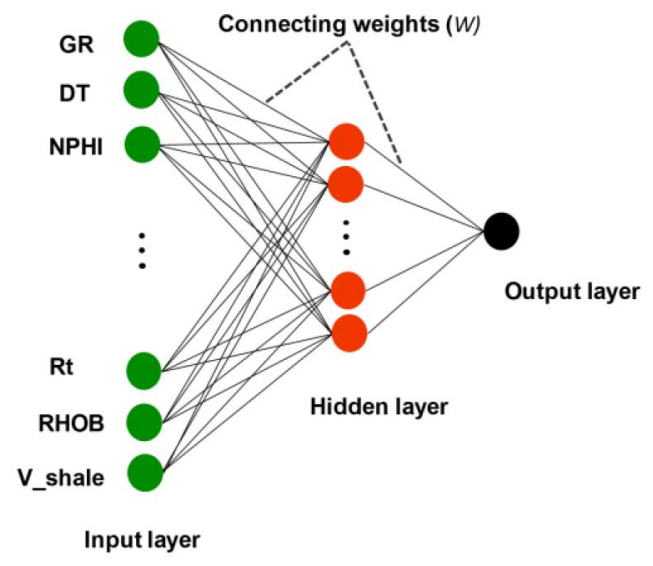

Figure 1-Structure of the designed three-layer neural network

$$
O^{*}=f\left[W_{o}+\sum_{j=1}^{N_{2}} W_{j} f_{j}\left(\omega_{0 j}+\sum_{i=1}^{N_{1}} \omega_{i j} I_{i}\right)\right]
$$

Here, $O^{*}$ and $I$ respectively are the output and input variables; $N_{l}$ is the dimension of the input vector and $N_{2}$ is the number of hidden neurons; $\omega_{i j}$ is the connection weight from $i$-th input neuron to the $j$-th hidden neuron and $W_{j}$ is the overall weight of $j$-th neuron; $\omega_{0 j}$ and $W_{0}$ are the related bias weights; and $f$ is the activation function (see Chen, 2006 for additional details). In this work all networks are trained with a linear activation function for the input layer and a tangent-sigmoid activation function for the hidden and output layers.

We also apply a bagging technique based on bootstrap sampling to generate multiple training sets from the original training database. The neural network would then be trained from each of these training sets (Breiman, 1994). Considering Figure 2, a bootstrap sample is constructed from a data set of dimension 
$\beta$ (with $\beta=1 \ldots N_{l}$ ), by randomly sampling from the space of $N_{l}$ original input variables (see (1)) with replacement (Step 1). Note that, due to replacement, some patterns may be selected more than once while some other patterns might not selected at all. It is expected that a bootstrap sample contains about $67 \%$ of the original data set patterns (Breiman, 1996). Next, each bootstrap data set is used to train a neural network (Step 2 in Figure 2). The predictions generated by each of these networks are then averaged to generate socalled bagged ANN ensemble predictions (Step 3 in Figure 2), which are expected to be associated with an improved degree of stability.

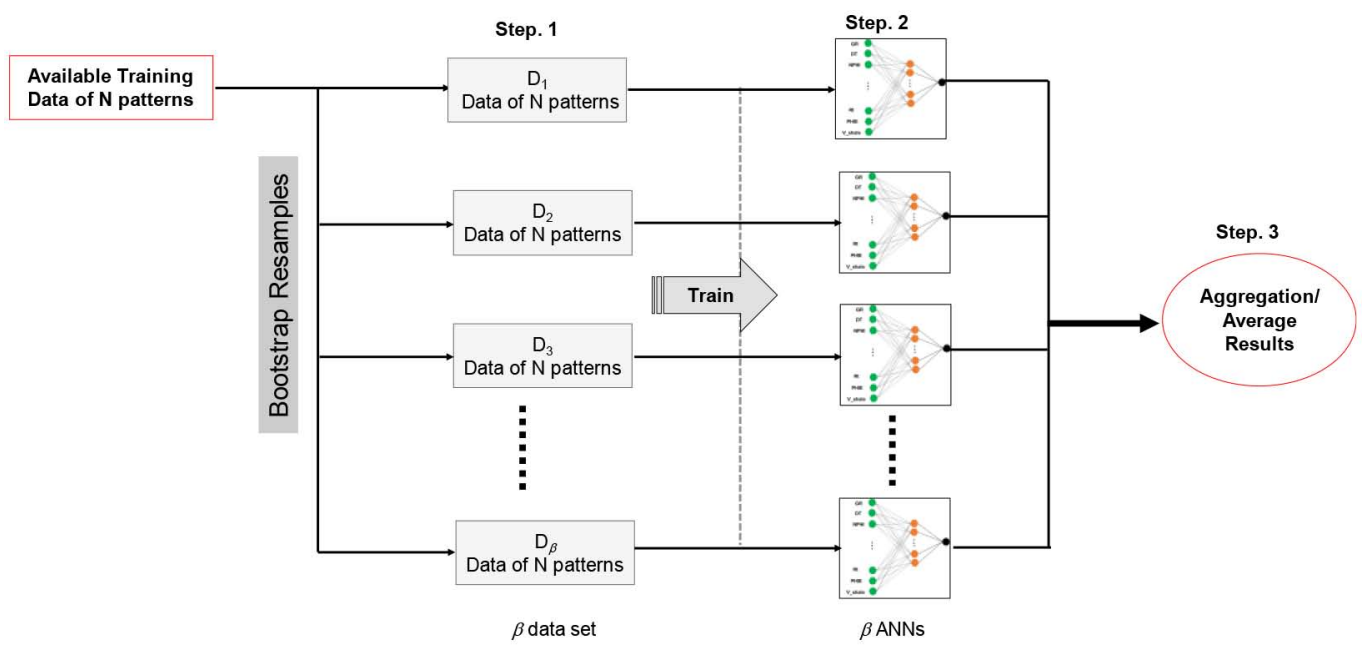

Figure 2-Schematic representation of a bagged ANN

\section{Results and discussion}

\section{Data processing}

The available dataset includes core and well log data. The core data comprise laboratory experiments (i.e., data on porosity and permeability) performed on diverse core samples taken from 11 wells between depths of 2482.59 and $2923.95 \mathrm{~m}$ (below ground level). Figure 3 depicts a scatterplot of porosity $(\phi)$ versus permeability $(\mathrm{K})$ data associated with the above mentioned laboratory experiments. The observed scattering of the data points is typically due to heterogeneity of the characteristics of the analyzed reservoir.

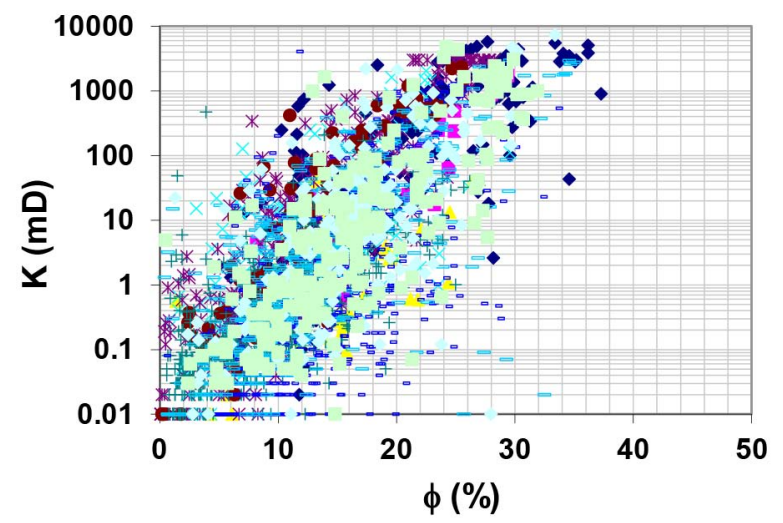

Figure 3-Scatterplot of porosity $(\phi)$ versus permeability $(K)$ data stemming from laboratory core experiments associated with 11 wells

Table 1 lists descriptive statistics of the data obtained from 3296 core samples extracted from 9 exploration wells. Our analysis reveal that porosity data are well interpreted by a Gaussian distribution 
(details not shown). The mild sample skewness might be due to the presence of clay in the reservoir rock texture. Observations of permeability are associated with an average value of $152.5 \mathrm{mD}$ and are positively skewed. These results indicate heterogenity of the reservoir characteristics, a result which is consistent with the geological make-up of the cores examined, mainly composed by crushed limestone and sandstone rocks.

Table 1-Descriptive statistics of porosity and permeability data obtained from core samples.

\begin{tabular}{|l|c|c|c|c|c|c|c|c|}
\hline & $\begin{array}{c}\text { Number of } \\
\text { observations }\end{array}$ & Mean & Median & Minimum & Maximum & Std.Dev. & Skewness & Kurtosis \\
\hline $\begin{array}{l}\text { Permeability } \\
(\mathbf{m D})\end{array}$ & 3296 & $152 / 49$ & 1.86 & 5 & 7240 & 542.32 & 5.47 & 36.52 \\
\hline Porosity (\%) & 3296 & $13 / 51$ & 12.8 & 3.50 & 37.21 & 6.95 & 0.46 & -0.16 \\
\hline
\end{tabular}

Well logging data are also collected from 8 wells for the analysis of porosity and permeability. These data include neutron porosity (NPHI), sonic logging (DT), gamma ray (GR), caliper (CALIP), density (RHOB), resistivity (Rt), well logging depth (Depth), laterolog deep (LLD), water saturation $\left(\mathrm{S}_{\mathrm{w}}\right)$ of origin rocks and dominant lithology such as shale (V-Shale). Table 2 lists descriptive statistics of these well log data. It can be seen that CALIP, Rt, RHOB and V-Shale are positively skewed with high Kurtosis, thus supporting a general picture of a heterogeneous reservoir.

Table 2-Statistical description of the well logging data.

\begin{tabular}{|c|c|c|c|c|c|c|c|c|}
\hline & $\begin{array}{c}\text { Observation } \\
\text { numbers }\end{array}$ & Mean & Median & Minimum & Maximum & Std.Dev. & Skewness & Kurtosis \\
\hline Depth & 3296 & 2689.1 & 2688.9 & 2482.59 & 2923.95 & 99.56 & -0.14 & -0.4 \\
\hline CALIP & 3296 & 7.92 & 8.37 & 5.63 & 22.19 & 2.27 & 1.63 & 4.6 \\
\hline Rt & 3296 & 0.34 & 0.17 & 0.052 & 7.08 & 0.52 & 4.35 & 2.86 \\
\hline DT & 3296 & 71.03 & 68.01 & 49.4433 & 133.69 & 12.22 & 0.41 & -1.03 \\
\hline GR & 3296 & 44.75 & 40.45 & 4.04 & 182.5 & 23.64 & 1.04 & 1.55 \\
\hline NPHI & 3296 & 0.18 & 0.18 & 0.05 & 0.58 & 0.08 & 1.14 & 3.21 \\
\hline LLD & 3296 & 0.12 & 0.12 & 0.03 & 0.38 & 0.08 & 0.16 & -0.84 \\
\hline RHOB & 3296 & 2.45 & 2.48 & 2.92 & 3.02 & 0.2 & -0.84 & 2.17 \\
\hline S & 3296 & 0.56 & 0.53 & 0.04 & 1 & 0.34 & 0.04 & -1.36 \\
\hline V-Shale & 3296 & 0.16 & 0.11 & 0.02 & 0.86 & 0.17 & 1.41 & 1.67 \\
\hline
\end{tabular}

Figure 4 depicts the bivariate correlation matrix between variables of the analyzed dataset. Porosity shows the highest correlatios with DT, RHOB, LLD, NPHI and $\mathrm{S}_{\mathrm{W}}$. Permeability displays the highest correlations with RHOP, DT, Depth, NPHI, Rt and $\mathrm{S}_{\mathrm{W}}$. 


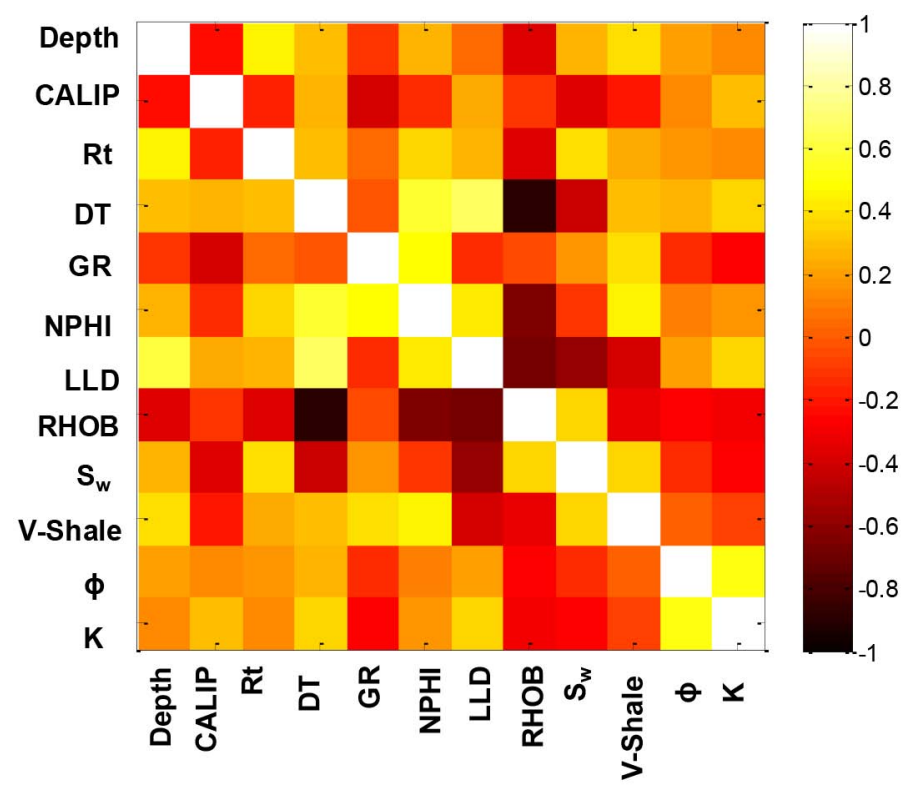

Figure 4-Bivariate correlation matrix of the analized dataset

\section{Principal component analysis (PCA)}

Principal Component Analysis (PCA) enables us to reduce the number of input variables for ANN models. Application of the method is well documented and esentially results in a new set of variables, which are defined as orthogonal principal components. It is commonplace to retain only the first few principal components which are needed to describe the total variance of the original data up to a given level of accuracy. The variance explained by each of the principal components is given in Figure 5 for porosity and permeability. These results suggest that the first 3 principal components explain almost $80 \%$ of the variability of either porosity or permeability due to variation of the input parameters.
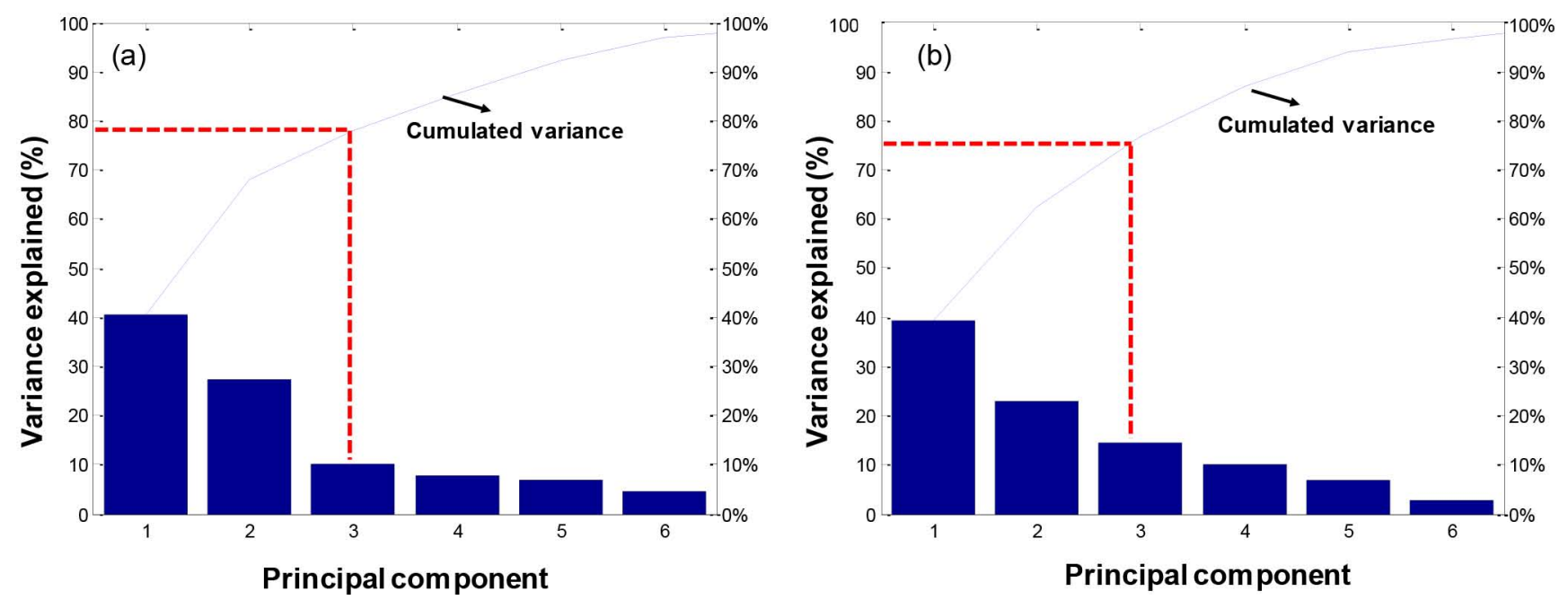

Figure 5-Percent variability explained by the first 6 principal components for (a) porosity $(\phi)$ and (b) permeability (K)

Coefficients associated with the original variables in the space of the first 8 principal components $\left(\mathrm{PC}_{\mathrm{i}}\right.$ with $\mathrm{i}=1, \ldots, 8)$ are listed in Table 3 . According to Table $3 \mathrm{a}$, the absolute values of the 3 largest coefficients in the space of the first three principal components $\left(\mathrm{PC}_{1}, \mathrm{PC}_{2}\right.$, and $\left.\mathrm{PC}_{3}\right)$ are mainly associated with $\mathrm{RHOB}$, NPHI, GR, $S_{w}$ and DT (see numbers in bold in Table $3 b$ ). This means that the first 3 principal components (which contribute to almost $80 \%$ of the variance of the output) are mainly given by a weighted mixture of 
these variables. A corresponding analysis is performed for permeability. As a result, one can observe that the the first three principal components are mainly associated with DT, GR, Depth, Rt, RHOB, NPHI, and $\mathrm{S}_{\mathrm{w}}$.

Table 3-Coefficients of the system parameters contributing to the first 8 principal components for (a) porosity (\#) and (b) permeability (K)

\begin{tabular}{|c|c|c|c|c|c|c|c|c|}
\hline \multicolumn{9}{|c|}{ (a) } \\
\hline \multirow{2}{*}{ Parameter } & \multicolumn{8}{|c|}{ Principal component } \\
\hline & $\mathrm{PC}_{1}$ & $\mathrm{PC}_{2}$ & $\mathrm{PC}_{3}$ & $\mathrm{PC}_{4}$ & $\mathrm{PC}_{5}$ & $\mathrm{PC}_{6}$ & $\mathbf{P C}_{7}$ & $\mathrm{PC}_{8}$ \\
\hline Rt & 0.073 & 0.184 & -0.104 & 0.090 & 0.436 & 0.154 & -0.296 & -0.024 \\
\hline RHOB & 0.495 & 0.090 & -0.325 & -0.090 & 0.142 & -0.592 & 0.364 & 0.485 \\
\hline GR & -0.028 & 0.507 & 0.112 & -0.418 & 0.613 & 0.098 & 0.012 & -0.192 \\
\hline NPHI & 0.389 & 0.252 & -0.046 & -0.177 & -0.364 & 0.660 & 0.106 & 0.346 \\
\hline DT & 0.170 & -0.041 & -0.380 & -0.683 & 0.197 & 0.261 & 0.560 & -0.477 \\
\hline Sw & -0.452 & -0.246 & 0.104 & 0.210 & 0.379 & 0.314 & 0.354 & 0.559 \\
\hline Depth & -0.241 & 0.123 & 0.061 & 0.437 & -0.258 & -0.116 & 0.571 & -0.262 \\
\hline LLD & 0.25 & -0.22 & -0.12 & -0.26 & 0.18 & -0.06 & 0.14 & -0.23 \\
\hline V-Shale & -0.18 & -0.14 & 0.25 & 0.02 & 0.16 & -0.21 & 0.7 & -0.03 \\
\hline CALIP & 0.15 & -0.08 & 0.19 & -0.21 & 0.23 & -0.01 & 0.20 & 0.06 \\
\hline$\phi$ & 0.25 & -0.22 & -0.88 & -0.26 & 0.18 & 0.02 & -0.06 & 0.001 \\
\hline
\end{tabular}

\begin{tabular}{|c|c|c|c|c|c|c|c|c|}
\hline \multirow{3}{*}{ Parameter } & \multicolumn{8}{|c|}{ (b) } \\
\hline & \multicolumn{8}{|c|}{ Principal component } \\
\hline & $\mathrm{PC}_{1}$ & $\mathrm{PC}_{2}$ & $\mathrm{PC}_{3}$ & $\mathrm{PC}_{4}$ & $\mathrm{PC}_{5}$ & $\mathrm{PC}_{6}$ & $\mathbf{P C}_{7}$ & $\mathrm{PC}_{8}$ \\
\hline Depth & -0.056 & 0.533 & -0.296 & -0.368 & 0.668 & -0.183 & -0.032 & 0.099 \\
\hline RHOB & -0.171 & 0.607 & 0.037 & -0.019 & -0.575 & -0.183 & -0.482 & 0.066 \\
\hline NPHI & -0.519 & 0.027 & 0.158 & -0.044 & 0.057 & 0.593 & 0.018 & 0.589 \\
\hline GR & 0.049 & 0.184 & 0.804 & 0.361 & 0.324 & -0.271 & -0.011 & 0.088 \\
\hline DT & -0.511 & -0.075 & -0.002 & -0.141 & -0.209 & -0.571 & 0.574 & 0.121 \\
\hline Sw & 0.524 & -0.091 & -0.166 & 0.062 & -0.107 & -0.253 & 0.014 & 0.781 \\
\hline LLD & 0.138 & 0.129 & 0.063 & 0.031 & -0.211 & 0.342 & 0.658 & -0.084 \\
\hline V-Shale & -0.01 & -0.16 & -0.13 & 0.21 & -0.13 & 0.29 & 0.22 & 0.24 \\
\hline CALIP & 0.15 & -0.05 & 0.12 & -0.18 & 0.21 & -0.09 & 0.17 & -0.06 \\
\hline Rt & -0.45 & -0.25 & -0.10 & -0.21 & -0.38 & 0.31 & 0.35 & -0.56 \\
\hline $\mathbf{K}$ & -0.213 & 0.144 & -0.456 & 0.841 & 0.123 & -0.026 & 0.052 & 0.003 \\
\hline
\end{tabular}

The results of a bivariate correlation analysis performed on the well logging variables selected through PCA are depicted in Figure 6. These results suggest that porosity is mainly correlated with DT, NPHI and GR and permeability is mainly correlated with GR, $\mathrm{S}_{\mathrm{w}}$, and DT and NPHI. 


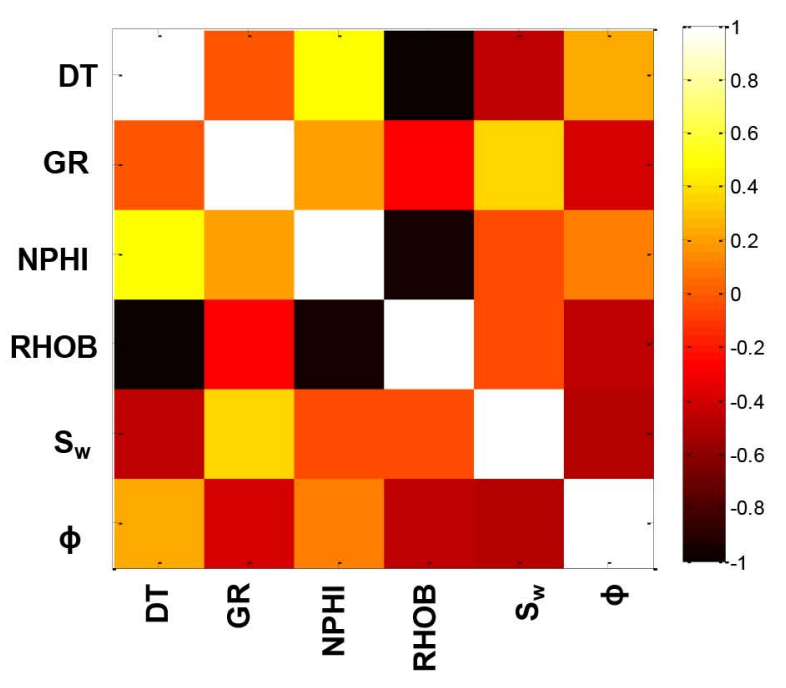

(a)

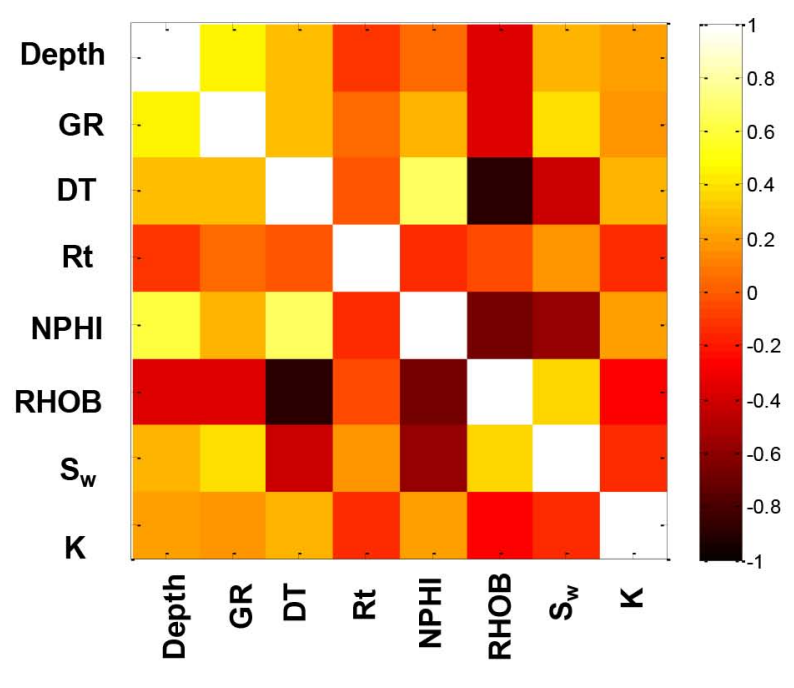

(b)

Figure 6-Correlation matrix for (a) porosity $(\varphi)$ and (b) permeability $(K)$ and the well log variables selected through PCA

\section{Artificial Neural Networks (ANNs)}

Input variables to ANNs comprise well logging data. Porosity and permeability data associated with the core samples from the same wells are taken as target outputs. A total of 3296 data points are employed to train, validate and test designed networks. The available dataset is divided into two categories. The first group comprises $65 \%$ of the total sampling points and is assigned to the process of network training and validation. Two third of these data points are use for training ANNs and the rest is employed for validation of the trained networks. An inadequate selection of data points for training ANNs may cause insufficient learning, and thus, poor ability of the network to provide reliable results. It is then critical that data selection for training networks ensure coverage of the entire available dataset. All data are normalized between 0.1 and 0.9 to improve the learning ability of the neural networks. This choice is due to the observation that tangent sigmoid activation function cannot attain zero or unit values.

Training ANNs aims at attaining the best possible correlation coefficient $\left(\mathrm{R}^{2}\right)$ and minimum MSE between predicted and observed values of prosity and permeability. The number of hidden layers and the corresponding number of neurons are varied to determine the best structure of the network leading to an optimized performance in reproducing the data. We compare training performance of the networks containig a number of hidden layers ranging between 1 and 3. It is found that satisfactory results are obtained with networks associated with a single hidden layer (details not shown). This is consistent with prior studies (e.g., Horink et al., 1989; Haykin, 1991; Bhatt et al., 2001) indicating a single hidden layer including enough neurons can be sufficient to cope with settings associated with a relatively high degree of complexity.

A scatterplot of the results constructed from a trained classical network yields values of $\mathrm{R}^{2}=73.7 \%$ and $72.2 \%$, respectively for porosity and permeability estimates.

Following the application of PCA, 5 and 6 well loging variables (see Figure 6) are respectively used as inputs to the networks for the assessment of porosity and permeability. Compared to the results from classical ANNs, training of PCA-based ANNs are associated with slightly improved values of $\mathrm{R}^{2}=79.8 \%$ and $74.1 \%$, respectively for porosity and permeability.

We then employ a bagging technique basd on bootstrap resampling to generate multiple training sets and use these to train an ensemble of ANNs (see Figure 2). The predictions generated by each network are averaged to generate more stable bagged ANN ensemble predictions. A number of 20 training data sets are generated on the basis of bootstrap resampling technique. These are provided to the networks according to the BPNN training technique. A configuration based on 9 input variables and 18 neurons in the 
hidden layer is selected as the optimum structure for the calculation of porosity. Such a network provides reliable performances for porosity (with $\mathrm{R}^{2}=94 \%$ at the validation stage). The same structure (with 10 input variables and 24 neurons in the hidden layer) is ranked as best for reproducing permeability data. Validation of the optimum network yield a value of $\mathrm{R}^{2}=85 \%$ to abservations of permeability. Table 4 lists results corresponding to the performance of optimum networks of different kinds in terms of correlation coefficent $\left(\mathrm{R}^{2}\right)$ and mean squer error (MSE).

Table 4-Validation performance of optimal neural networks for reproduction of porosity, $\varphi$, and permeability, $\mathrm{K}$

\begin{tabular}{|c|c|c|c|c|c|c|}
\hline \multirow{2}{*}{ Type of neural network } & \multicolumn{2}{|c|}{$\begin{array}{c}\text { Optimal network geometry } \\
\text { (input-hidden-0utput lyers) }\end{array}$} & & \multicolumn{2}{c|}{ MSE } \\
\cline { 2 - 7 } & $\phi$ & K & $\phi$ & K & $\phi(\%)$ & K(mD) \\
\hline Classical neural network & $9-22-1$ & $10-24-1$ & $73.7 \%$ & $72.2 \%$ & 0.0089 & 0.0074 \\
\hline PCA neural network & $5-20-1$ & $7-20-1$ & $79.8 \%$ & $74.1 \%$ & 0.0085 & 0.0092 \\
\hline Bagged neural network & $9-22-1$ & $10-24-1$ & $94 \%$ & $85 \%$ & $\mathbf{0 . 0 0 1}$ & 0.0034 \\
\hline
\end{tabular}

Finally, we employ the optimum bagged NNs to predict porosity and permeability in 3 wells which are not used in the calculations and where incomplete well logging information is available. Figures 7 and 8 respectively illustrate the prediction results of porosity and permeability based on optimzed bagging ANN for these three wells. Solid lines correspond to the bagged neural network predictions, core data being represented by circles. As one can observe, there is a genrally good agreement between values predicted from optimum bagged ANNs with data of porosity and permeability.
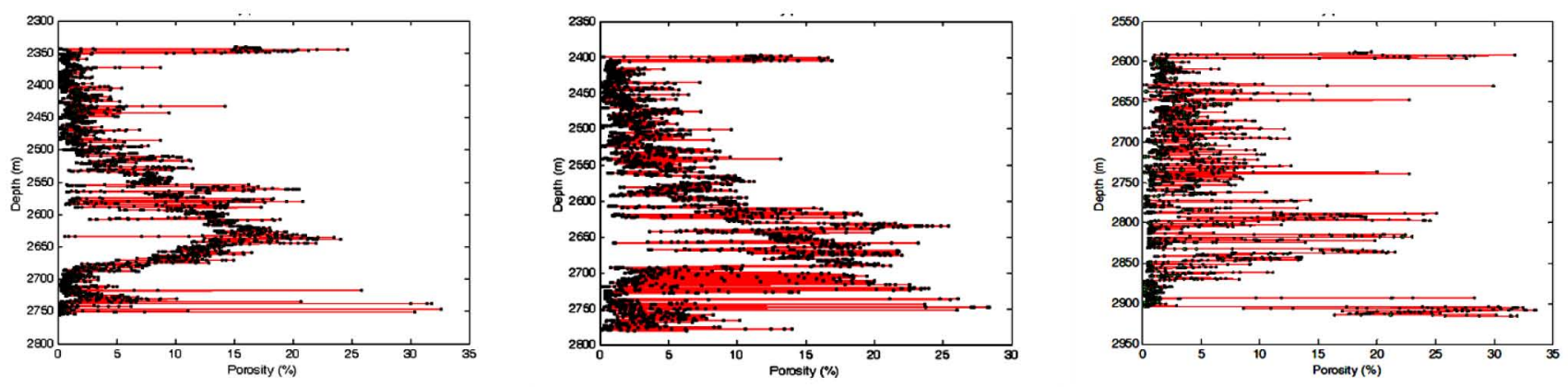

Figure 7-Prediction of porosity from optimum bagged ANN for the wells with incomplete well logging data
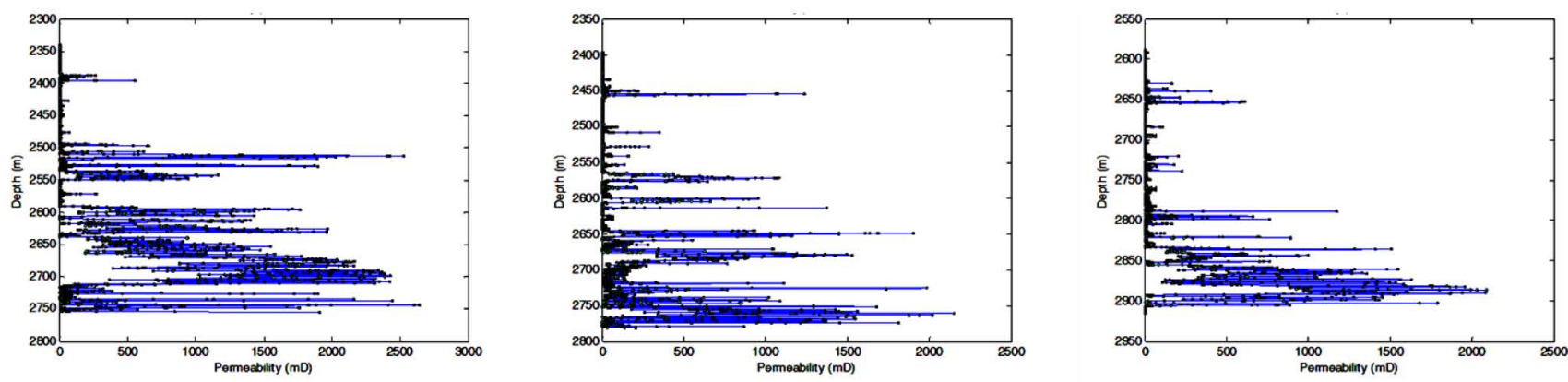

Figure 8-Prediction of permeability from optimum bagged ANN for the wells with incomplete well logging data 


\section{Conclusions}

Our work leads to the following major conclusions:

- The main scope of this work was to show the advantage of relying on intelligent methods (i.e., artificial neural network) to estimate permeability and porosity using some available laboratory core data and well logging information.

- Results show that ANNs can be a viable and computationally cheap solution for dealing with incomplete set of petrophysical information, their implementation being not hampered in the absence of a complete characterization of the geological model of a reservoir.

- Principal Component Analysis (PCA) suggests that it is possible to reduce the dimensionality of the input variables. It is then observed that PCA based neural network models are characterized by a slightly improved performance in comparison with the classical neural networks.

- A trained bagged neural network provides results of improved quality as compared against those obtained from both PCA based and classical neural network models.

\section{References}

Aminian, K., and Ameri, S. 2005. Application of artificial neural networks for reservoir characterization with limited data. J. Petrol. Sci. Eng. 49 (3): 212-222. doi: 10.1016/j.petrol.2005.05.007

Bhatt, A., Helle, H.B., and Ursin, B. 2001. Application of committee machines in reservoir characterization while drilling: a novel neural network approach in log analysis. Proc. 6th Nordic Symposium on Petrophysics, Trondheim, Norway, 15-16 May.

Bowden, G. J. 2003. Forecasting water resources variables using artificial neural techniques. Doctoral thesis, University of Adelaide, Australia.

Breiman, L. 1994. Bagging predictors. Machine Learning 24 (2): 123-140. doi: 10.1023/A:1018054314350

Chen, C.H. 2006. Committee-machine-based models for permeability prediction. Doctoral thesis, National Cheng Kung University of Taiwan, Taiwan.

Coates, G.R. and Dumanoir, J.L. 1973. A new approach to improved log-derived permeability. Proc. SPWLA 14th Annual Logging Symposium, Lafayette, Louisiana, 15-16 May.

Gibbs, M. S., Morgan, N., Maier, H. R., Dandy, G. C., Nixon, J. B. and Holmes, M. 2006. Investigation into the relationship between chlorine decay and water distribution parameters using data driven methods. Mathematical and Computer Modelling 44(5-6): 485-498. doi: 10.1016/j.mcm.2006.01.007

Hagan, M.T., and Menhaj, M. 1994. Training feed forward networks with the Marquardt algorithm. IEEE Trans. Neural Netw. 5 (6): 989-993. doi: 10.1109/72.329697

Haykin, S. 1999. Neural Networks: A Comprehensive Foundation. Second edition, Prentice-Hall, USA.

Helle, H.B., Bhatt, A. and Ursin, B. 2001. Porosity and permeability prediction from wireline logs using artificial neural networks: a North Sea case study. Geophysical Prospecting 49 (4): 431-444. doi: 10.1046/j.1365-2478.2001.00271.x

Hornik, K., Stinchcombe, M. and White, H., 1989. Multilayer feedforward networks are universal approximators. Neural Networks 2 (5): 359-366. doi: 10.1016/0893-6080(89)90020-8

Huang Z., Shimeld J., Williamson M. and Katsube J. 1996. Permeability prediction with artificial neural network modelling in the Venture gas field, offshore eastern Canada. Geophysics 61 (2): 422-436. doi: 10.1190/1.1443970

Huang Z. and Williamson M.A. 1997. Determination of porosity and permeability in reservoir intervals by artificial neural network modelling, offshore eastern Canada. Petroleum Geoscience 3: 245-258. doi: 10.1144/petgeo.3.3.245

Johnson, W.W. 1994. Permeability determination from well logs and core data. Presented in Permian Basin Oil and Gas Recovery Conference, Texas, 14-18 March. SPE-27647-MS. doi: 10.2118/27647-MS

Ligtenberg, J.H. and Wansink, A.G. 2001. Neural network prediction of permeability in the El Garia formation, Ashtart oilfield, offshore Tunisia. Journal of Petroleum Geology 24 (4): 389-404. doi: 10.1111/j.1747-5457.2001.tb00682.x

Lim, J.S. 2005. Reservoir properties determination using fuzzy logic and neural networks from well data in offshore Korea. J. Petrol. Sci. Eng. 49 (3): 182-192. doi: 10.1016/j.petrol.2005.05.005

Mohaghegh, S.D. 2005. Recent developments in application of artificial intelligence in petroleum engineering. J. Petrol Technol. 57(4): 86-91. SPE-89033-JPT. doi: 10.2118/89033-JPT

Mohaghegh, S. 2000. Virtual-intelligence applications in petroleum engineering: Part 1-Artificial neural networks. $J$. Petrol Technol. 52(9): 64-73. SPE-58046-JPT. doi: 10.2118/58046-JPT 
Olsson, J., Uvo, C.B., Jinno, K., Kawamura, A., Nishiyama, K., Koreeda, N., Nakashima, T. and Morita, O. 2004. Neural networks for rainfall forecasting by atmospheric downscaling. Journal of Hydrologic Engineering 9(1): 1-12. doi: 10.1061/(ASCE)1084-0699(2004)9:1(1)

Ranaee, E., Porta, G., Riva, M. and Guadagnini, A. 2014, Investigation of saturation dependency of oil relative permeability during WAG process through linear and non-linear PCA. Proc. ECMOR XIV-14th European Conference on the Mathematics of Oil Recovery, Catania, Italy, 11-18 September. doi: 10.3997/2214-4609.20141800

Silpngarmlers, N., Guler, B., Ertekin, T. and Grader, A.S. 2002. Development and testing of two-phase relative permeability predictors using artificial neural networks. SPE J. 7(3): 299-308. SPE-79547-PA. doi: 10.2118/79547-PA

Tamhane, D., Wong, P.M., Aminzadeh, F. and Nikravesh, M. 2000. Soft computing for intelligent reservoir characterization. Proc. SPE Asia Pacific conference on integrated modelling for asset management, Yokohama, 2526 April. SPE-59397-MS. doi: 10.2118/59397-MS

Wong, P.M., Jang, M., Cho, S. and Gedeon, T.D. 2000. Multiple permeability predictions using an observational learning algorithm. Computers and Geosciences 26(8): 907-913. doi: 10.1016/S0098-3004(00)00026-1 